\title{
Referencias anatómicas para la tenolisis percutánea de los flexores de la mano
}

\author{
M. Brotat, C. Simón, B. García Medrano, L. García Flórez, M. A. Martín Ferrero \\ Servicio de Cirugía Ortopédica y Traumatología. Hospital Clínico Universitario de Valladolid. Universidad de Medicina de \\ Valladolid. España.
}

Resumen: Objetivos: La tenosinovitis estenosante de los flexores de la mano es una patología muy frecuente en el ámbito laboral. Se han descrito distintos tratamientos de tipo conservador, aunque el tratamiento definitivo de los casos que no responden a otros tratamientos es quirúrgico, siendo la técnica abierta y la liberación percutánea las dos técnicas de elección. Esta última ha obtenido resultados superiores de satisfacción aunque la ausencia de visualización incrementa las lesiones neurovasculares y el riesgo de apertura incompleta de la polea, por ello es necesario establecer puntos de referencia anatómicos para realizar una correcta liberación.

Material y método: Se realizó un estudio experimental en cinco manos de cadáver en el Departamento de Anatomía de la Universidad de Valladolid. Se realizaron mediciones de las longitudes de los dedos y de la polea. Se registró la posición de la aguja y de cada uno de los dedos al realizar la polectomía percutánea y se comprobó si la sección de la polea Al fue completa, si existió alguna laceración de los tendones o si se produjo una lesión iatrógena del paquete neurovascular o de la polea $\mathrm{A} 2$.

Resultados: Para establecer el punto de entrada de la aguja intramuscular se tomó como referencia la intersección entre el eje del dedo y el surco dígito-palmar. En el caso del tercer y cuarto dedo la entrada fue en el punto de la intersección, mientras que en el resto se desplazó I-I,5 mm en dirección medial.

El ángulo de entrada de la aguja intramuscular en el plano anterior osciló entre 0-30 grados en función del dígito, mientras que en el plano lateral fue siempre 0 excepto en el cuarto dedo que corresponde a un valor de -60 grados respecto al eje palmar. No se observó ninguna lesión iatrógena de los tendones flexores, del paquete neurovascular o de la polea A2. La apertura fue completa en todos los dedos salvo en un caso del segundo dedo.

Conclusión: La tenolisis percutánea de la polea $\mathrm{Al}$ es una alternativa a la liberación abierta tradicional, aunque existe controversia debido a la relación riesgo-beneficio. El conocimiento de las referencias anatómicas así como la posición exacta del dígito y de los instrumentos utilizados ayudan a evitar la liberación incompleta de la polea, los problemas neurovasculares y la lesión de estructuras adyacente.

En el presente estudio anatómico se ha comprobado que es una técnica segura ya que no ha ocurrido ninguna lesión iatrógena y fiable, que se ha conseguido una liberación completa en todos los casos salvo uno.

Palabras claves: referencias anatómicas, polectomía percutánea, dedo en resorte, estudio en cadáver.

\begin{abstract}
Abastract: Purpose:Triger finger is a common pathology in the workplace. There have been reported different conservative treatments for its treatment but the definitive treatment is ussualy surgical and it may be through an open or percutaneous technique The last has earned higher satisfaction scores although the absence of viewing increases the risk of neurovascular injury or of incomplete opening of the pulley, making necessary to set the anatomical landmarks for proper release. Material and method:An experimental study was performed in five cadaver hands at the University of Valladolid Department of Anatomy. Measurements of lengths of digits and the pulley were performed. The position of the needle and the finger while making the polectomy were recorded and the complete section of the Al pulley, the injury of the flexore tendons, the neurovascular bundle or the A2 pulley were check.

Results: To set the entry point of the intramuscular needle the intersection between the axis of the digit-finger and palmar groove was used as a reference. For the third and fourth finger the entry point was in the intersection point while for the rest of fingers was placed I-I,5 mm more medial.

The entrance angle of the needle in the anterior plane was between 0-30 degrees depending on the digit, while in the lateral plane was always 0 except for the fourth finger which corresponds to a value of -60 degrees to the volar axis. There was no injury in the flexor tendons, the neurovascular bundle or the A2 pulley. There was a complete release of all the pulleys except one case of the second finger.

Conclusion: Percutaneous tenolysis of AI pulley is an alternative to traditional open release although there is controversy because of the risk-benefit ratio. Knowledge of the anatomical landmarks and the exact position of the digit and the tools used to help prevent incomplete release of the pulley, neurovascular problems and injury of adjacent structures. In the currente study it has been proven that is a safe technique as there has been no iatrogenic injury and reliable as a complete pollectomy has been obtain in all fingers except
\end{abstract} one.

Key words: Anatomical landmarks, percutaneous trigger finger reléase, trigger finger, cadaver study.

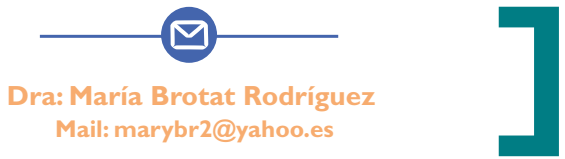


El dedo en resorte representa una de las patologías más frecuentes de la mano. El tratamiento conservador es de primera elección aunque si éste falla es preciso recurrir a la cirugía 1,2.

La técnica abierta ofrece buenos resultados aunque algunas complicaciones, como los problemas derivados de la cicatriz, han promovido el desarrollo de la técnica percutánea',3.

Muchos estudios han demostrado que se trata de un proceso seguro, eficaz y rentable económicamente. Sin embargo, la ausencia de visualización directa, la posibilidad de liberación incompleta de la polea Al y el riesgo de daño de las estructuras neurovasculares hacen que sean necesarios estudios en cadáver para establecer las referencias anatómicas que permitan un resultado óptimo.

Lorthoir, en 1958, fue el primero que describió la tenolisis percutánea utilizando el pliegue palmar proximal como referencia del tercer, cuarto y quinto dedo 3 . Entre 1970 y 1985 varios autores como Barton ${ }^{4}$, Schneider y Hunter ${ }^{5}$ establecieron la relación existente entre el borde de las poleas y las articulaciones metacarpofalángicas ${ }^{3}$.

En los años 90, Nagoshi y Lyu', basándose en las referencias de Lorthoir establecieron la localización del borde proximal de la polea $\mathrm{Al}$ en función del dedo, afirmando que se encuentra localizado a $5 \mathrm{~mm}$ distal del pliegue palmar proximal del segundo dedo y a pocos milímetros del pliegue palmar distal en el resto. En un estudio más reciente, Jongjirasiri afirma que el borde proximal de la polea se encuentra en el punto central de la cabeza del metacarpiano ${ }^{7}$.

El objetivo de nuestro estudio es establecer las referencias anatómicas para la realización de la técnica percutánea.

\section{MATERIALY MÉTODO}

Se realizó un estudio experimental practicando la disección anatómica de cinco manos de cadáver en el Departamento de Anatomía de la Universidad de Valladolid.

Se obtuvieron mediciones de la longitud y anchura de cada uno de los dedos, identificando la posición de la polea Al y A2, midiéndolas y observando su relación con los pliegues palmares y digitales. Se usaron agu-

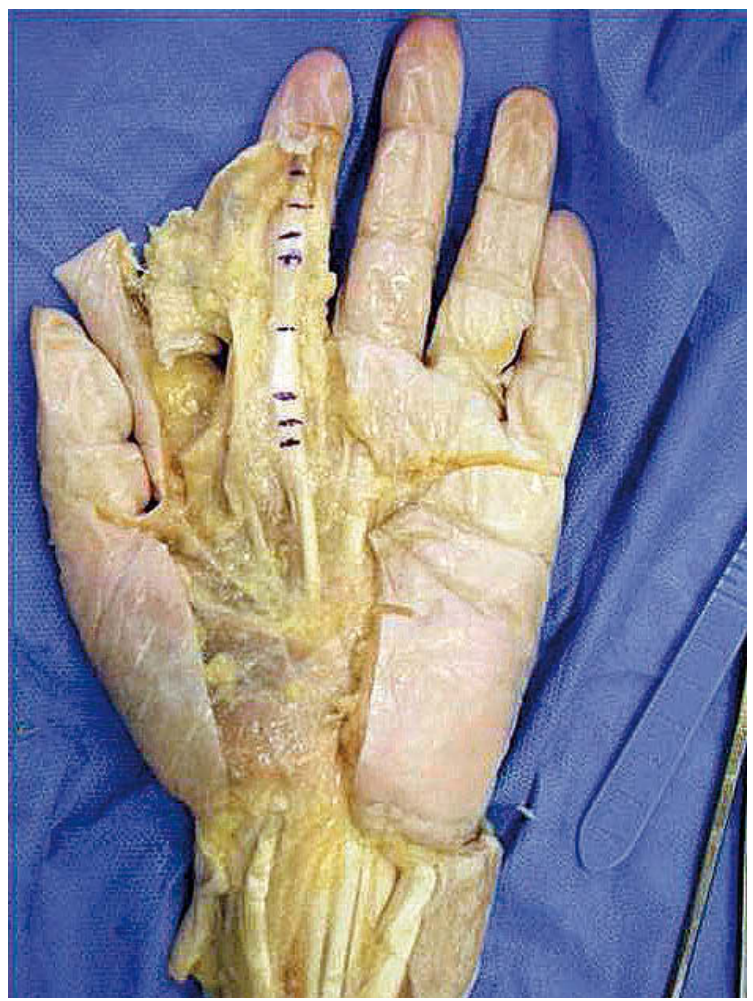

Figura I. Medición de longitudes de poleas.

jas de Kirschner (AK) y agujas de $21 \mathrm{G}$ a través de las poleas Al para indicar la dirección de la polectomía. (2) Figura I.

Para realizar la liberación percutánea se situó la aguja en la vaina del tendón flexor a nivel de la polea Al orientando el bisel de forma lateral. Mediante un movimiento longitudinal y paralelo a la vaina del tendón se seccionó dicha polea. La punción se realizó exactamente sobre la línea media del metacarpiano. El dedo en resorte representa una de las patologías más frecuentes de la mano. El tratamiento conservador es de primera elección aunque si éste falla es preciso recurrir a la cirugía',2.

Por último se comprobó si la sección de la polea fue completa, si existió alguna laceración de los tendones o si se produjo una lesión iatrógena del paquete neurovascular o de la polea A2. () Figura 2.

\section{RESULTADOS}

Las longitudes de los dedos se encontraron en un rango entre $55 \mathrm{~mm}$ y $77 \mathrm{~mm}$. La longitud media de la polea AI fue de $5.4 \mathrm{~mm}$ y la polea A2 de $14.5 \mathrm{~mm}$. 


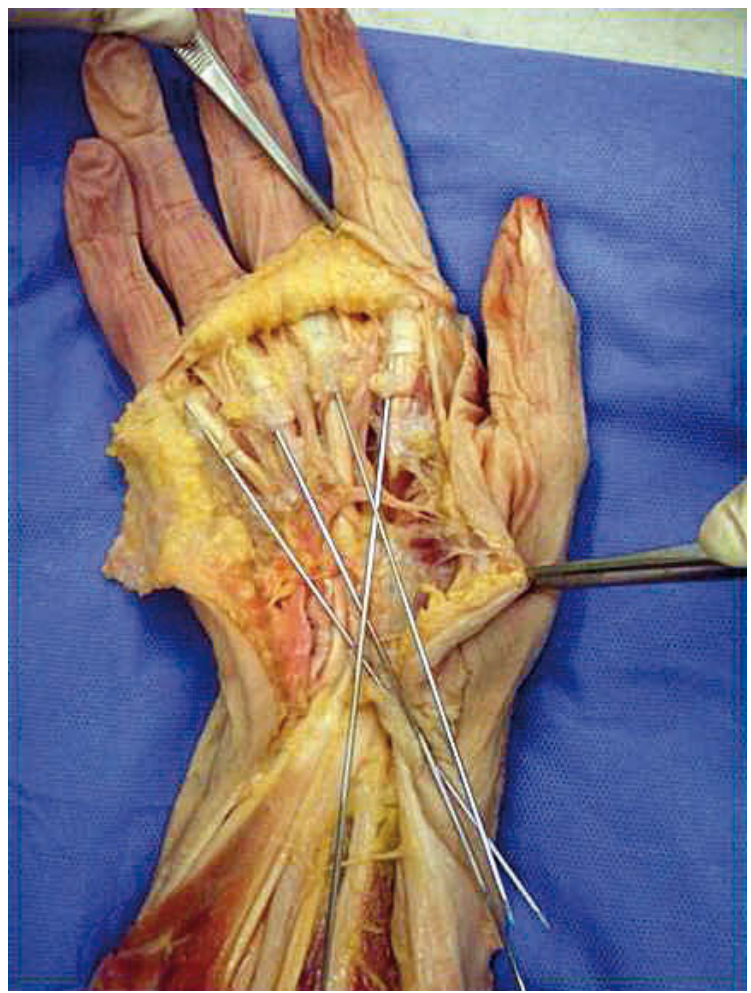

Figura. 2. AK insertadas en vainas de los flexores para observar la dirección y ángulo correcto.

con una distancia entre ambas que osciló entre $4.6 \mathrm{~mm}$ y $5,4 \mathrm{~mm}$.

\section{Primer dedo}

El primer dedo es uno de los más conflictivos por la cercanía de los tendones flexores al paquete neurovascular. En la disección anatómica se observó que la distancia media de los tendones al nervio colateral cubital fue de $4,8 \mathrm{~mm}$, mientras que la distancia al nervio colateral radial fue de $1 \mathrm{~mm}$ ( Figura 3 .

El punto de entrada se situó en el pliegue distal coincidiendo con la cabeza del metacarpiano. Es importante destacar que cuanto más proximal sea el punto de entrada existe más riesgo de lesionar las ramas nerviosas.

El dedo se colocó en extensión completa manteniendo una hiperextensión de la falange distal. El ángulo de la aguja intramuscular fue de treinta grados en el plano anterior.

Al realizar la disección tras la aplicación de la técnica percutánea no se observó daño en la polea A2 ni en el paquete vasculonervioso, no existieron laceraciones tendinosas y la liberación de los tendones fue completa en todos los casos.

\section{Segundo dedo}

La distancia media desde el borde del tendón flexor al nervio colateral radial fue de $3 \mathrm{~mm}$ y al nervio colateral cubital de 1,6 $\mathrm{mm}$. Al realizar la tenolisis percutánea hay que tener especial cuidado con la rama colateral cubital ya que cruza cerca de la polea AI.

El punto de entrada de la aguja se situó $5 \mathrm{~mm}$ distal del pliegue palmar proximal (siguiendo las referencias establecidas por Lorthioir), posición que coincide con el centro de la cabeza del metacarpiano.

El dígito debe se colocó en extensión completa y el ángulo de entrada osciló entre los 20-30 grados.

Al realizar la disección tras la aplicación de la técnica percutánea no se observó daño en la polea $\mathrm{A} 2$ ni en el paquete vasculonervioso. No existieron laceraciones tendinosas aunque se observó un caso de liberación incompleta (6.66\% del total).

\section{Tercer dedo}

En el tercer dedo la distancia media de los tendones flexores a los nervios colaterales radial y cubital fue de $2,2 \mathrm{~mm}$ y $2,6 \mathrm{~mm}$ respectivamente.

El punto de entrada de la aguja se situó a pocos milímetros del pliegue palmar distal con una angulación de treinta grados y el dedo en extensión completa.

Al realizar la disección tras la aplicación de la técnica percutánea no se observó daño en la polea $\mathrm{A} 2$ ni en el paquete vasculonervioso, no existieron laceraciones tendinosas y la liberación de los tendones fue completa en todos los casos.

\section{Cuarto dedo}

En el cuarto dedo la distancia de los tendones flexores a los nervios colateral radial y cubital fue de $1.8 \mathrm{~mm}$.

Al igual que en el tercer dedo el punto de entrada se situó a pocos milímetros del pliegue palmar distal coincidiendo con la cabeza del metacarpiano. En este caso la posición del dígito fue en hiperextensión, con un ángulo de entrada de la aguja de menos 60 grados respecto al eje palmar. (2) Figura 4.

Al realizar la disección anatómica tras la aplicación de la técnica percutánea no se observaron laceraciones tendinosas, daño en la polea A2 ni en el paquete vas- 

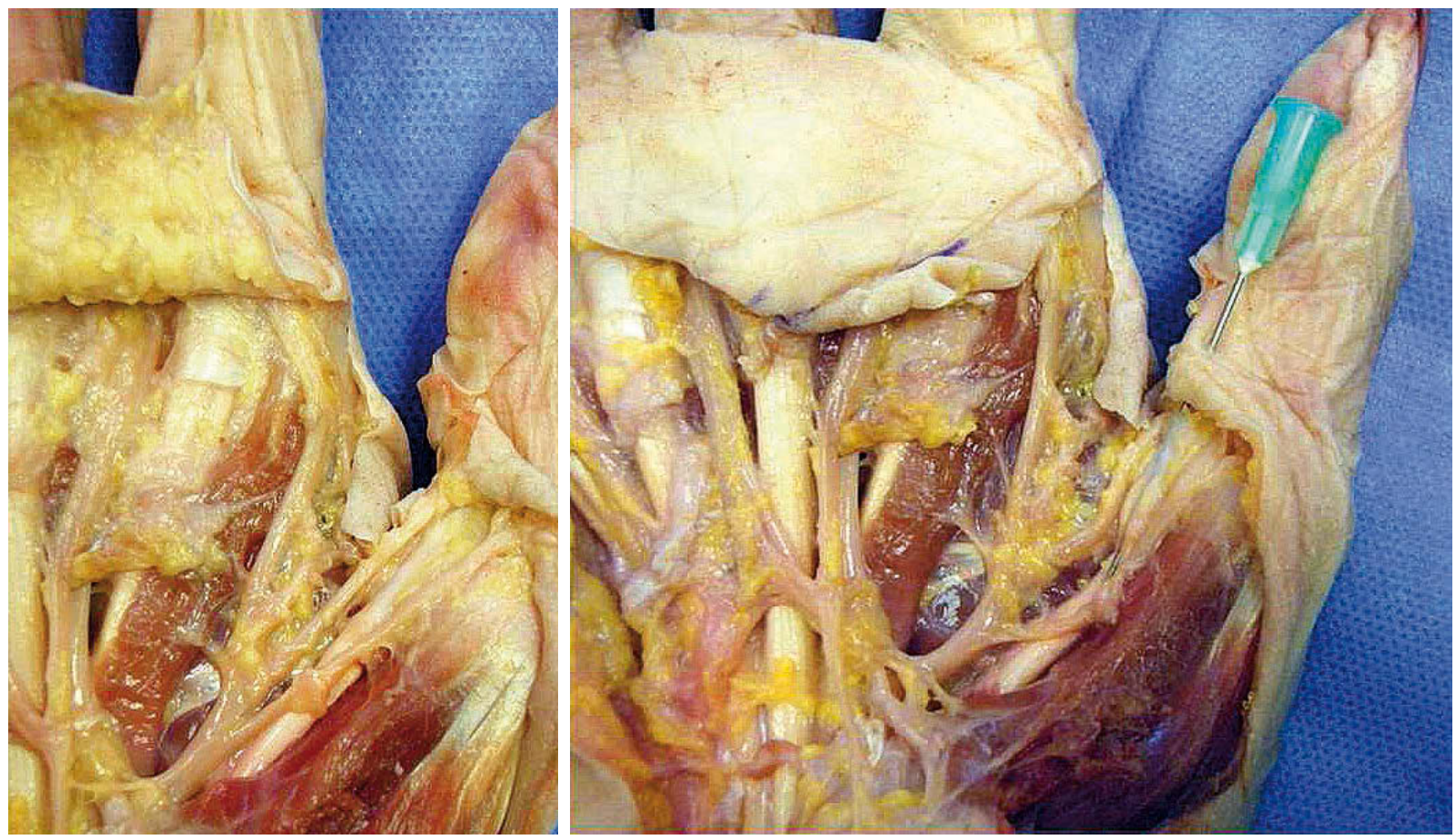

Figura 3. Estrecha relación de los tendones flexores con el paquete vasculonervioso en el primer dedo.

culonervioso, y la liberación de los tendones fue completa en todos los casos.

\section{Quinto dedo}

En el quinto dedo destacó la amplia distancia de los tendones flexores al nervio colateral cubital $6 \mathrm{~mm}$ mientras que la distancia media al nervio colateral radial fue de I,4 mm,

El punto de entrada de la aguja fue a 2-3 milímetros proximal al pliegue palmar distal (siguiendo las referencias de Lorthoir), el dedo se colocó en extensión completa @ Figura 5.

Al realizar la disección anatómica tras la aplicación de la técnica percutánea no se observaron laceraciones tendinosas, daño en la polea $\mathrm{A} 2$ ni en el paquete vasculonervioso, y la liberación de los tendones es completa en todos los casos.

\section{DISCUSIÓN}

Muchos estudios describen la liberación percutánea de la polea $\mathrm{Al}$ en el tratamiento del dedo en resorte. Aunque Lorthoir estableció de forma general la localización del borde proximal de la polea $\mathrm{Al}^{3}$, en la práctica la polea empieza unos milímetros más distales de las marcas establecidas ${ }^{2.8}$. Por ello, muchos autores (Barthon, Schneider, Hunter) prefieren utilizar la relación con la articulación metacarpofalángica para evitar las variaciones étnicas y antropométricas ${ }^{4,5}$.

La liberación incompleta, el riesgo de lesionar la A2 y el posible daño neurovascular son las posibles complicaciones que se han analizado en el presente trabajo.

En varios estudios realizados en cadáver se ha observado una liberación incompleta de la polea Al sobre

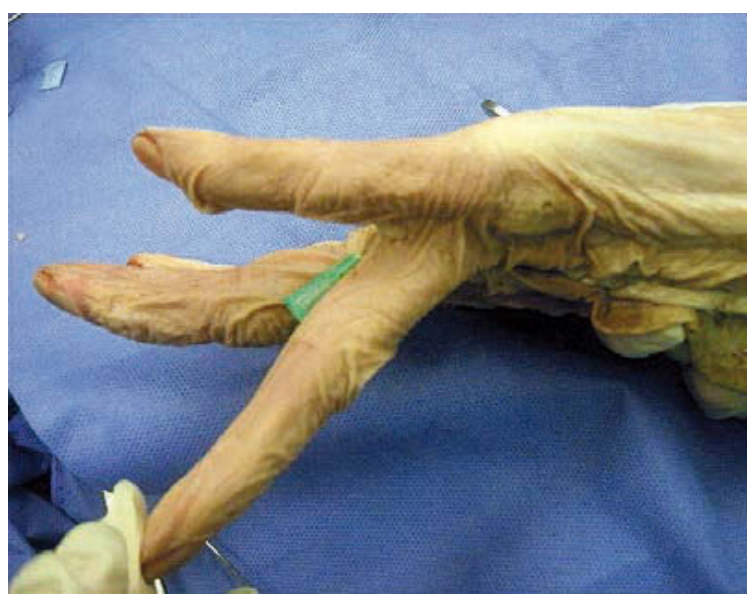

Figura 4. Hiperextensión del cuarto dedo para la realización de la técnica percutánea. 


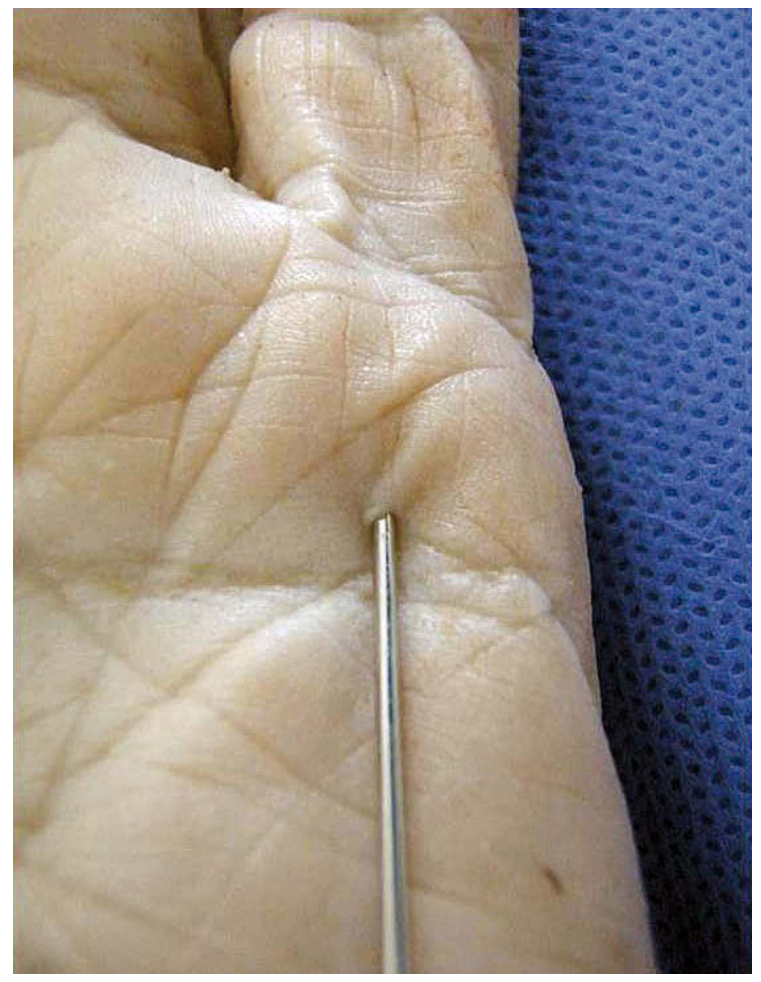

Figura 5. Punto de entrada de la aguja a pocos milímetros proximales al pliegue palmar distal situando el dígito en extensión completa.

todo en su porción más proximal. Bain observa un 32\% en su estudio, un porcentaje muy alto respecto al observado en nuestra serie. Bain afirma que, en pacientes vivos, es más sencillo realizar la liberación completa de la polea ya que habitualmente existe un nódulo que nos permite identificar mejor la entrada de la aguja9. Sin embargo, Hazani describió un 100\% de liberación completa de los tendones ${ }^{8}$.

En diversos estudios se obervan porcentajes que oscilan entre el 60 y el $80 \%$ de laceraciones de los tendones sin llegar a rotura completa. Sin embargo, en otros más actuales el porcentaje disminuye a un $26 \%$, debido posiblemente a una mayor precisión en las referencias anatómicas y una mejor orientación de la aguja ${ }^{10}$.

El hecho de utilizar una aguja estrecha provoca que sean necesarios varios cortes para obtener una liberación completa del tendón aumentando el riesgo de laceraciones en los tendones ${ }^{10,11}$. En nuestro estudio no se ha encontrado ninguna lesión de los tendones flexores.

La valoración del estado de la polea A2 es fundamental. Aunque en nuestro estudio no se ha observado afectación de la misma, en estudios previos existe una incidencia de un 22\% de lesión, afectando una media de 2.3 milímetros de la polea A2. Se cree que es debido a la posición de entrada más distal de la aguja". Sin embargo, es difícil realizar estudios clínicos que valoren la afectación de la polea A2 ya que en la mayoría de los casos no existe sintomatología".

Uno de los mayores riesgos es la lesión del paquete neurovascular ya que la visualización directa no es posible. Estudios previos afirman que el mayor riesgo es en el primer, segundo y quinto dedos $1,9 \cdot \operatorname{Sin}$ embargo, en nuestra práctica clínica la técnica percutánea se realiza fundamentalmente en el primer dedo sin observar lesiones neurovasculares.

Es importante destacar que los cadáveres, en muchas ocasiones, tienen las marcas alteradas y el tejido varía debido a los fluidos postmortem, además de no presentar nódulos en las poleas, de forma que puede sesgar los resultados 11,12 .

En los últimos años algunos autores como Chern y Jou apuestan por el concepto de liberación percutánea extravaginal, cortando la polea desde la superficie de la vaina sinovial hacia tendón flexor, guiada por ecografía ${ }^{13}$.

\section{CONCLUSIÓN}

La tenolisis percutánea de la polea $\mathrm{Al}$ es una alternativa a la liberación abierta tradicional, aunque existe controversia debido a la relación riesgo-beneficio. El conocimiento de las referencias anatómicas así como la posición exacta del dedo y de los instrumentos utilizados ayudan a evitar la liberación incompleta de la polea, los problemas neurovasculares y la lesión de estructuras adyacente.

En el presente estudio anatómico se ha comprobado que es una técnica segura ya que no ha ocurrido ninguna lesión iatrógena y fiable que se ha conseguido una liberación completa en todos los casos salvo uno.

\section{AGRADECIMIENTOS}

Nuestro agradecimiento al Dr. Juan Francisco Pastor Vázquez, Profesor titular del Departamento de Anatomía de la Universidad de Valladolid por el apoyo y la ayuda prestada. 


\section{CONFLICTOS DE INTERESES}

Los autores declaran no tener conflictos de intereses.

\section{BIBLIOGRAFÍA}

I. Paulius KL, Maguina P.Ultrasound-Assisted Percutaneous Trigger Finger Release: Is it Safe? Hand (NY). 2009:4:35-7.

2. Chaves Moreno A. Tenosinovitis estenosante del tendón flexor. Medicina legal de Costa Rica. 2008;25(I):59-67.

3. Lorthoir J. Surgical treatment of trigger-finger by a subcutaneous method. J Bone Joint Surg Am. 1958;40;793-5.

4. Barton NJ. Experimental study of optimal location of flexor tendon pulleys. Plast Reconstr Surg. 1969;43:125-9.

5. Schneider LH,Hunter JM. Flexor tendons late reconstruction. En: Green PD (Ed). Operative Hand Surgery. New York: Churchill Livingstone; 1982. P.I 375-40.

6. Nagoshi M, Hashizume H, Nishida K, Takagoshi H, $\mathrm{Pu}$ J, Inoue $\mathrm{H}$. Percutaneous release for trigger finger in idiopathic and hemodialysis patients. Acta Med Okayama. 1997;5 I (3): I55-8.
7. JongjirasiriY.The results of percutaneous release of trigger digits by using full handle knife 15 degrees: an anatomical hand surface landmark and clinical study.J Med Assoc Thai. 2007;90(7): 1 348-55.

8. Dunn MJ, Pess GM. Percutaneous trigger finger release: a comparison of a new push knife and a 19-gauge needle in a cadaveric model. J Hand Surg Am. 1999;24(4):860-5.

9. Bain Gl, Turnbull J, Charles MN, Roth JH, Richards RS. Percutaneous AI pulley release: a cadaveric study. J Hand Surg Am. 1995;20(5):78I -4.

10. Gordon J, Lindsay BS, Gordon L. Surface markers for locating the Pulleys and flexor tendon anatomy in the palm and fingers with reference to minimally invasive incisions. J Hand Surg Am. 20 I2;37(5): 9|3-8.

I I. Habbu R, Putnam MD, Adams JE. Percutaneous release of the AI pulley: a cadaver study. J Hand Surg Am. 20 12;37( I I):2273-7.

12. Fiorini HJ, Santos JB, Hirakawa CK, Sato ES, Faloppa F, Albertoni WM. Anatomical study of the AI pulley: length and location by means of cutaneous landmarks on the palmar surface. J Hand Surg Am. 20। I:36(3):464-8.

13. Jou IM, Chern TC. Sonographically assisted percutaneous release of the al pulley: a new surgical technique for treating trigger digit. J Hand Surg $\mathrm{Br}$. 2006;3। (2): 19|-9. 